\title{
RECHERCHES SUR LA MÉTHODE \\ DE MESURE DE LA DIGESTIBILITÉ \\ D'UN ALIMENT \\ CONDITIONS D’EMPLOI DE CETTE MÉTHODE
}

PAR

A. GASNIER et J. P. VACHEL

Laboratoire de l'Institut professionnel de contrôle et de recherches

des industries de l'alimentation animale, Paris

\section{PLAN DU MEMOIRE}

\section{Technique expérimentale et protocole des essais.}

Digestibilité de la ration de référence en fonction de sa proportion dans le régime.

a) Digestibilité des rations totales.

b) Digestibilité de la ration de référence.

Dịscussion.

Digestibilité d'un aliment incorporé en porportions variables à une ration de référence.

Discussion : a) Digestibilité du son.

b) Digestibilité du tourteau de soja.

Expériences de WATSON et al. (Ottawa).

Digestibilité des différents composants de deux mélanges : aliment commercialson et aliment commercial-tourteau de soja.

a) Mélange aliment commercial-son.

b) Mélange aliment commercial-tourteau de soja.

Digestibilité des différents composants d'un aliment ajouté au régime de base.

a) Erreurs possibles sur la valeur des coefficients de digestibilité.

b) Conditions d'emploi de la méthode de Kellner.

c) Erreurs dues à l'analyse chimique.

Conclusions.

La méthode classique de détermination de la digestibilité d'un aliment (méthode de KELLNER) consiste à mélanger cet aliment, en proportions déterminées, à une ration dite de référence dont on connaît l'utilisation digestive 
lorsqu'on la fournit seule aux animaux. Un tel mode de mesure suppose implicitement que la digestibilité de la ration de référence reste invariable quelles que soient les proportions du mélange.

Nous avons cherché à vérifier, chez le Lapin, l'exactitude de cette hypothèse. Utilisant des mélanges en proportions variables de deux aliments, nous avons calculé, à l'aide de systèmes de 2 équations à 2 inconnues, la digestibilité de la ration de référence et celle d'un aliment qu'on lui ajoute.

D'autre part, à l'aide des mêmes données, employées cette fois selon la méthode de KELINER, nous avons déterminé la digestibilité, également chez le Lapin, d'un aliment incorporé en proportions variables à une ration de référence. Ce nouveau calcul nous a permis de comparer les résultats obtenus à partir des deux méthodes utilisées.

Enfin, grâce au matériel analytique ainsi accumulé, nous avons tenté de déterminer la digestibilité des différents constituants alimentaires des rations utilisées et ce travail nous a permis de préciser les conditions d'emploi de la méthode de KELLNER.

\section{TECHNIQUE EXPÉRIMENTALE ET PROTOCOLE DES ESSAIS}

Les animaux, lapins adultes de race pure Chinchilla ou Blanc de Vendée, sont placés dans des cages spéciales, décrites par l'un de nous (I939), cages qui permettent une détermination aussi précise que possible des ingesta et des excreta. A chaque changement de régime, les lapins sont soumis à une adaptation de 7 à ro jours de durée et les mesures proprement dites sont poursuivies pendant Io jours. Les cages sont placées dans une pièce dont la température peut être approximativement réglée en hiver $\left( \pm 2^{\circ}\right)$ à l'aide d'un chauffage élcetrique d'appoint.

Les animaux reçoivent leur alimentation une fois par jour, le matin entre 9 h 30 et Io h, sous forme d'une " pâtée " imbibée d'eau. Au cours de la distribution dans les mangeoires, on prélève plusieurs échantillons qui sont recueillis dans une capsule et mis à sécher à l'étuve à roo ${ }^{\circ}$ L'ensemble de ces échantillons nous parâ̂t représenter aussi exactement que possible la composition moyenne de la ration distribuée tout au long d'une période expérimentale.

Chaque jour également, les refus et les excreta sont recueillis et pesés à $0,2 \mathrm{~g}$ près. Les fèces sont mises à sécher à l'étuve à roo et, une fois séchées, stockées jusqu'au moment de l'analyse. Les techniques d'analyse des différents constituants des rations et des excreta sont celles décrites dans la Brochure : Législation des Aliments des Animaux (juin r948) publiée par les Organisations Professionnelles de 1'Alimentation Animale. Cependant, nous avons dû modifier la méthode de détermination des matières grasses dans les fèces, les résultats que nous obtenions avec la méthode employée pour les aliments n'étant pas reproductibles avec une approximation suffisante ; aussi n'avons-nous pu déter- 
miner les digestibilités des matières grasses et de l'extractif non azoté que pour nos deux dernières séries (Séries $\mathrm{IX}$ et $\mathrm{X}$ - Annexe $\mathrm{V}$ ). Cette modification fera l'objet d'une communication spéciale.

Nous avons systématiquement vérifié que l'analyse de l'échantillon global de nourriture, constitué par l'ensemble des prélèvements effectués chaque jour sur la ration distribuée aux animaux, fournit des valeurs très voisines de celles que l'on peut calculer à partir de la composition centésimale des constituants du régime. Par exemple, nous avons trouvé, pour le régime de la série IV de nos essais, la composition suivante, pour roo de matière sèche :

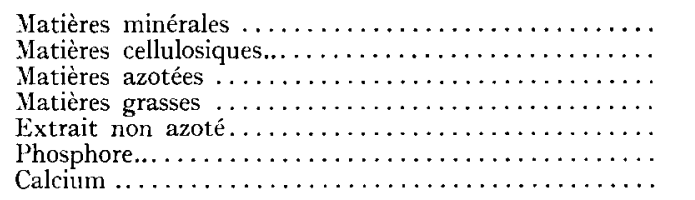

\begin{tabular}{|c|c|c|}
\hline 6,96 & & $7,28)$ \\
\hline $9,5^{2}$ & ") & $9,80)$ \\
\hline 20,45 & ) & $: 19,77)$ \\
\hline 3,45 & " & $4, \mathrm{TO})$ \\
\hline 59,62 & $n$ & : 59,05$)$ \\
\hline $\mathrm{I}, \mathrm{O}$ & $"$ & $1,02)$ \\
\hline 1,0 & n & $1,05)$ \\
\hline
\end{tabular}

Nous avons choisi comme ration de référence, un aliment composé du commerce (Farine $\mathrm{n}^{\circ} \mathrm{I}$ ) ; à cette ration, nous avons incorporé, en proportions variables, soit un son de blé, soit un tourteau de soja. Les compositions de ces aliments sont données en Annexe (Annexe I).

Nous avons réalisé sur les lapins Chinchilla, 4 séries de mesures suivant le schéma ci-dessous :

\begin{tabular}{|c|c|c|}
\hline No des séries & $\%$ de farine & $\%$ de son \\
\hline$\overline{\text { II }}$ & - & - \\
\hline III & $\begin{array}{l}90 \\
85\end{array}$ & 15 \\
\hline IV $\ldots \ldots \ldots \ldots \ldots \ldots \ldots$ & 80 & 20 \\
\hline$V \ldots \ldots \ldots \ldots \ldots \ldots$ & I00 & o \\
\hline
\end{tabular}

(Avant que commence l'adaptation au régime de la série II, les animaux ont été progressivement habitués à leur nouvelle alimentation et à leur nouvel habitat pendant 30 jours.)

Et, sur 5 lapins Blanc de Vendée, 3 séries de mesures d'après le protocole suivant :

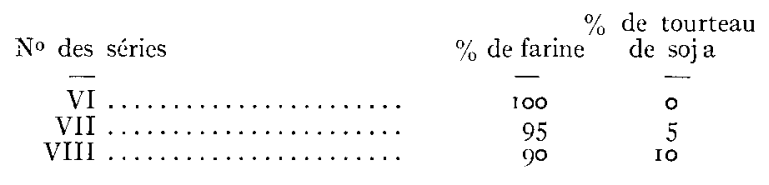

\section{DIGESTIBILITÉ DE LA RATION DE RÉFÉRENCE EN FONCTION DE SA PROPORTION DANS LE RÉGIME}

L'expérimentation fournit directement les valeurs de la digestibilité de la matière sèche de la ration totale et c'est à partir de ces valeurs que l'on peut déterminer l'utilisation digestive des composants du régime. On trouvera, dans le tableau I ci-dessous, les résultats d'ensemble que nous avons obtenus dans les diverses séries expérimentales. Les éléments du calcul de ces résultats sont donnés en Annexes (Annexes II et III). 


\section{TABLEAU I}

Digestibilités \% brutes - moyennes et individuelles - des mélanges son-farine ${ }^{\circ} 1$ et soja-farine $n^{\circ} 1$

I Mélange son-farine.

\begin{tabular}{|c|c|c|c|c|c|c|}
\hline \multirow{2}{*}{ Séries } & \multirow{2}{*}{$\%$ de son } & \multicolumn{4}{|c|}{ Numéros des lapins } & \multirow{2}{*}{$\begin{array}{c}\text { Moyennes } \\
\mathbf{M}\end{array}$} \\
\hline & & 2 & 3 & 4 & 5 & \\
\hline V.. & o & $74,0 \pm 3,2$ & $83,4 \pm 3,0$ & $7 \mathrm{I}, 7=\mathrm{I}, \mathrm{I}$ & $72,1 \pm 3,3$ & $74,6 \ldots$ \\
\hline II .. & IO & $73,2 \pm 2,8$ & $76,0 \pm 3,6$ & $72,8 \div \mathrm{I}, 8$ & $71,4 \pm 1,4$ & $73,0 \pm 1,4$ \\
\hline III $\ldots$ & I 5 & $72,1 \div 4,2$ & $74,9+5,6$ & $68,6 \pm \mathrm{I}, 5$ & $70,8 \pm 2,6$ & $71,2 \pm 1,8$ \\
\hline IV ... & 20 & $72,4 \pm 2,4$ & $70,9 \pm 4,8$ & $70,4 \div 0,9$ & $70,0 \pm 1,4$ & $70,9 \pm 1,4$ \\
\hline
\end{tabular}

20 Mélange soja-farine $n^{0} 1$.

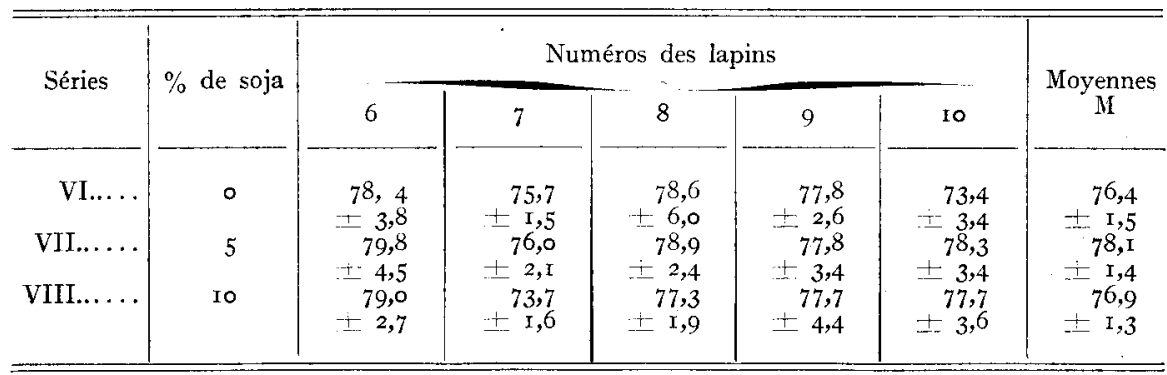

Notre projet initial comportait, pour le tourteau de soja, la réalisation d'une quatrième série expérimentale, le tourteau devant être ajouté à la ration de référence à raison de $7,5 \%$. Malheureusement, nous n'avons pas pu nous procurer le même aliment commercial que celui qui nous avait servi pour les essais précédents, et nous avons dû étudier en outre la digestibilité de la nouvelle farine (aliment $n^{0} 2$ - Annexe I) qui nous a été fournie. Ce sont les résultats de ces deux dernières séries que l'on trouvera dans le tableau I bis ci-dessous.

\section{TABLEAU I bis}

Digestibilités $\%$ brutes - moyennes et individuelles - du mélange soja-farine $n^{\circ} 2$

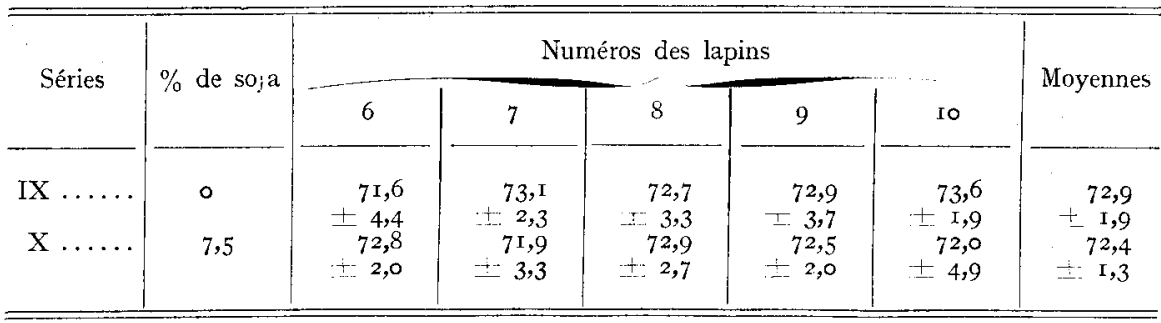

(1) Les digestibilités individuelles moyennes qui figurent dans les tableaux de ce mémoire sont obtenues à partir des ingesta et excreta totaux de ro jours et non pas par la moyenne aritmétique des ıo digestibilités quotidiennes. Les digestibilités moyennes $\mathbf{M}$ correspondant à chacune des séries expérimentales sont obtenues de la même façon. De plus, chacune des moyennes des tableaux I est suivie de son erreur standard. 


\section{a) - Digestibilité des rations totales}

Les résultats groupés dans le tableau I nous permettent de constater : $x^{\circ}$ que la digestibilité moyenne décroît quand le pourcentage de son dans la ration augmente ; toutefois, les différences entre les valeurs prises deux à deux ne sont pas significatives. (Pour les séries V et IV entre lesquelles existe la plus grande différence, $t=\mathrm{I}, 73$. Signification pour $t$ au moins égal à $\mathrm{I}, 99$.) Il est donc normal que nous ayons constaté, au cours de ces essais que le poids moyen des animaux n'a pas cessé d'augmenter lentement. On remarquera en outre que l'influence du son dans 1'alimentation se traduit très différemment d'un animal à 1'autre.

$2^{\circ}$ Que 1'addition, jusqu'à xo $\%$, de tourteau de soja à une farine commerciale pour Lapins ne modifie pas, en moyenne, la digestibilité de la ration totale. Les différences enregistrées, qu'il s'agisse de 1'une ou de l'autre des rations de base que nous avons utilisées, ne sont pas significatives.

$3^{\circ}$ Que la différence de digestibilité moyenne de l'aliment $n^{\circ}$ I entre les deux races de lapins sur lesquelles nous avons expérimenté $(74,6 \%$ pour les "Chinchilla " — Série V - et 76,4 \% pour les "Blanc de Vendée " - Série VI) n'est pas significative $(t=0,78)$.

\section{b) Digestibilité de la ration de référence}

A l'aide des données précédentes, nous pouvons calculer les digestibilités des deux composants principaux du régime : aliment de référence et son ou tourteau de soja. Il suffit pour cela, d'écrire que la digestibilité de la ration totale est la somme pondérée des digestibilités des deux composants en question. Soient $x$ et $y$ leurs digestibilités respectives, on peut écrire :

$$
\mathrm{A} x+\mathrm{B} y=\mathrm{D}
$$

en appelant $\mathrm{D}$ la digestibilité pour roo de la ration totale. Si nous groupons nos séries d'essais 2 à 2 , nous pourrons calculer les valeurs $x$ et $y$ des digestibilités dans les différentes conditions où nous nous sommes placés.

Ce mode de calcul revient en fait à supposer, comme on le fait dans la méthode classique, que les digestibilités $x$ et $y$ ne varient pas lorsque change la composition de la ration totale ; mais il a l'avantage, d'une part, de ne rien inférer en ce qui concerne la digestibilité du régime de référence, d'autre part, de limiter la validité de l'hypothèse selon laquelle les digestibilités ne varient pas, à des variations de faible amplitude de la composition des rations.

Les résultats des calculs que nous avons pu effectuer sont réunis dans le tableau II. 
TABIEAU II

Digestibilités \% calculées - moyennes et individuelles - de la ration de référence

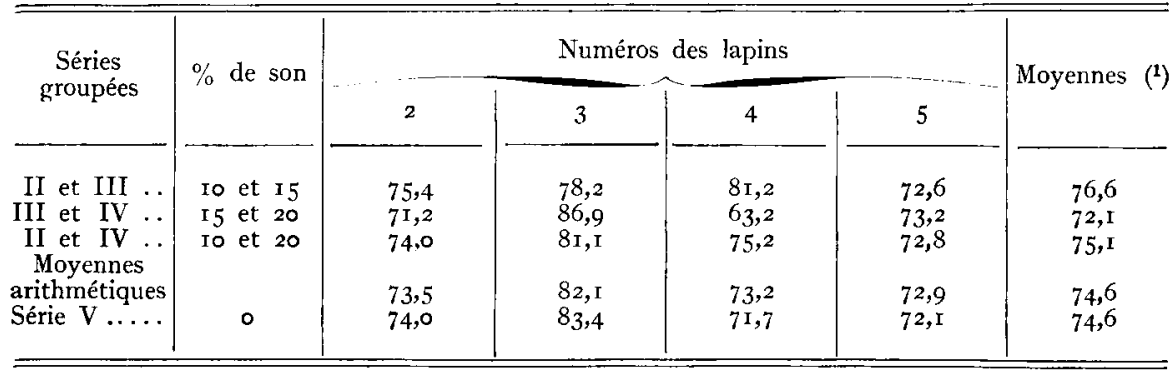

\begin{tabular}{|c|c|c|c|c|c|c|c|}
\hline \multirow{2}{*}{$\begin{array}{l}\text { Séries } \\
\text { groupées }\end{array}$} & \multirow{2}{*}{$\%$ de soja } & \multicolumn{5}{|c|}{ Numéros des lapins } & \multirow{2}{*}{ Moyennes } \\
\hline & & 6 & 7 & 8 & 9 & Io & \\
\hline $\begin{array}{l}\text { VII et VIII } \\
\text { Série VI .. }\end{array}$ & $\begin{array}{c}5 \text { et } \text { ro } \\
\text { o }\end{array}$ & $\begin{array}{l}80,6 \\
78,4\end{array}$ & $\begin{array}{l}78,3 \\
75,7\end{array}$ & $\begin{array}{l}80,5 \\
78,6\end{array}$ & $\begin{array}{l}77,9 \\
77,8\end{array}$ & $\begin{array}{l}78,9 \\
73,4\end{array}$ & $\begin{array}{l}79,3 \\
76,4\end{array}$ \\
\hline
\end{tabular}

(') Calculées d'après les valeurs $\mathrm{M}$ du tableau $\mathrm{I}$.

\section{Discussion}

Io Les résultats obtenus au cours des essais sur la digestibilité du son montrent que :

Les digestibilités moyennes de la ration de référence semblent décroître quand le pourcentage de son augmente, mais ces différences ne sont pas significatives. ( $t=0,7$ I pour la plus grande différence, signification pour $t$ supé rieur à 2,45 .)

La digestibilité moyenne déterminée dans les essais de la série $\mathrm{V}$ (farine seule) étant de 74,6 alors qu'elle est de 76,6 d'après les résultats combinés des séries II et III, on pourrait penser au phénomène de digestibilité associative du mélange aliment-son. Mais la différence entre ces 2 valeurs n'est pas significative. ( $t=\mathrm{I}$,OI - Signification pour $t$ supérieur à 2,02.)

Il est intéressant de remarquer en outre que la moyenne arithmétique des 3 valeurs moyennes calculées par cette méthode, soit 74,6, est exactement celle que nous avons trouvée expérimentalement. (Série $V-$ tableau I) ; ce qui confirme la non signification des différences entre les résultats calculés.

Il semble donc, au moins dans les conditions de la présente expérimentation, que la digestibilité de la ration de référence ne varie pas, en moyenne, lorsqu'on ajoute à cette ration des quantités croissantes de son.

$2^{0}$ En ce qui concerne les valeurs calculées à partir des deux séries d'essais réalisées sur le tourteau de soja, il semblerait que la digestibilité de la ration de référence soit légèrement supérieure à celle de la même ration distribuée 
seule. On pourrait alors penser - comme précédemment pour le son - au phénomène de digestibilité associative du mélange aliment-soja. Mais la différence entre les deux valeurs moyennes de digestibilité de la ration de référence, avec et sans soja $-79,3$ et $76,4 \%$ n'est pas significative $\left(t=2, \mathbf{I}_{5}\right.$ Signification pour $t=2,3 \mathrm{I}$ ).

Par conséquent, dans les conditions de nos essais, nous sommes en droit de penser que le tourteau de soja, incorporé à l'aliment de référence, ne fait pas varier la digestibilité de la matière sèche de celui-ci.

$3^{\circ}$ Les résultats individuels confirment dans l'ensemble les conclusions que nous avons pu tirer de l'étude des moyennes. Il y a lieu toutefois de noter que chez certains animaux - lapins no 3 et 4 - on enregistre de grosses variations des valeurs de la digestibilité de la ration de référence, bien que les valeurs moyennes calculées pour chaque lapin soient très voisines de celles qui ont été déterminées directement (Série V). De tels résultats sont bien difficiles à interpréter ; tout au plus confirment-ils qu'il est indispensable d'expérimenter sur le plus grand nombre possible d'animaux pour obtenir des valeurs de signification certaine.

\section{DIGESTIBILITÉ D'UN ALIMENT INCORPORÉ EN PROPORTIONS VARIABLES A UNE RATION DE RÉFÉRENCE}

Nous pouvons maintenant essayer de comparer les valeurs trouvées pour la digestibilité du son ou pour celle du tourteau de soja à l'aide de la méthode classique de KELLNER et à l'aide du calcul que nous avons défini précédemment. Les éléments de cette comparaison sont groupés dans le tableau III.

TABLEAU III

a) Digestibilité du son ( $p .100$ secs)

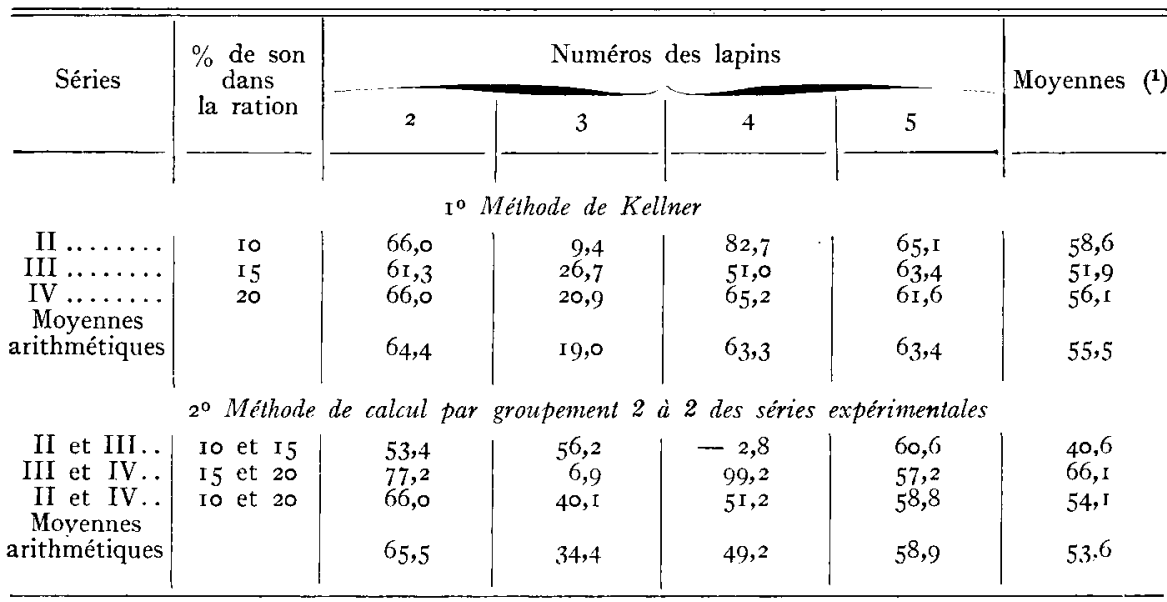

(1) Calculées d'après les valeurs $\mathrm{M}$ du tableau $\mathrm{I}$. 
b) Digestibilité du tourteau de soja ( $p .100$ secs)

\begin{tabular}{|c|c|c|c|c|c|c|c|}
\hline \multirow{2}{*}{ Séries } & \multirow{2}{*}{$\begin{array}{c}\% \text { de soja } \\
\text { dans } \\
\text { la ration }\end{array}$} & \multicolumn{5}{|c|}{ Numéros des lapins } & \multirow[t]{2}{*}{ Moyennes } \\
\hline & & 6 & 7 & 8 & 9 & Io & \\
\hline \multirow{3}{*}{$\begin{array}{r}\text { VII...... } \\
\text { X...... } \\
\text { VIII...... } \\
\text { Moyennes } \\
\text { arithmét. }\end{array}$} & \multirow{3}{*}{$\begin{array}{c}5 \\
7,5 \\
\text { I0 }\end{array}$} & \multicolumn{5}{|c|}{ Io Méthode de Kellner } & \multirow{3}{*}{$\begin{array}{c}110,4 \\
66,3 \\
81,4 \\
86,0\end{array}$} \\
\hline & & $\begin{array}{r}106,4 \\
87,6 \\
84,4\end{array}$ & $\begin{array}{l}8 \mathrm{I}, 6 \\
57, \mathrm{r} \\
55,7\end{array}$ & $\begin{array}{l}84,6 \\
75,3 \\
65,6\end{array}$ & $\begin{array}{l}77,8 \\
67,6 \\
76,8\end{array}$ & $\begin{array}{r}171,4 \\
57,6 \\
116,4\end{array}$ & \\
\hline & & 92,8 & & & $74, \mathrm{I}$ & I I $5, \mathrm{i}$ & \\
\hline \multicolumn{8}{|c|}{$2^{\circ}$ Méthode de calcul par groupement 2 a 2 des séries expérimentales } \\
\hline VII et VIII & 5 et 10 & 64,6 & 32,3 & 45,5 & 75,9 & 66,9 & $5.5,3$ \\
\hline
\end{tabular}

\section{DISCUSSION}

\section{a) Digestibilité du son}

Io La détermination de la digestibilité du son par la méthode de KELINER donne des résultats individuels extrêmement dispersés — de 9,4 à 82,7\% alors que les valeurs calculées à partir des moyennes du tableau I - et qui groupent l'ensemble des mesures effectuées sur les 4 animaux - ne varient qu'entre $5 \mathrm{I}, 9$ et $58,6 \%$. De plus, si 1'on considère chacune des séries expérimentales, les moyennes des 4 valeurs individuelles (pour autant qu'un tel calcul ait une signification) ne sont pas statistiquement différentes.

(Séries II et III : $t=0,29$.- Séries III et IV $: t=0,20$. - Séries II et IV : $t=0, \mathrm{I}_{3}$. - Signification pour $t=2,45$.)

$2^{\circ}$ Le mode de calcul que nous avons expérimenté conduit à des résultats encore plus dispersés ; la digestibilité du son varie, chez le même animal de $-2,8 \%$ (?) à 99,2\%. Ces valeurs n'ont évidemment aucun sens physiologique.

$3^{\circ}$ Il est cependant remarquable de constater que, si l'on se contente de n'enregistrer que les moyennes arithmétiques calculées sur l'ensemble des résultats, les 2 modes de détermination mènent à des résultats tout à fait comparables : 55,5 et $53,6 \%$. C'est une nouvelle confirmation du fait que les mesures de digestibilité doivent être réalisées sur le plus grand nombre possible d'animaux.

\section{b) Digestibilité du tourteau de soja}

Les valeurs que nous fournit la méthode de KELLNER sont, ici encore, extrêmement dispersées ; certaines d'entre elles dépassent même largement roo \%,ce qui nous paraît dû uniquement au mode de calcul utilisé. Les moyennes 
arithmétiques que nous avons calculées, uniquement dans un but comparatif, n'ont évidemment aucune valeur physiologique.

De plus, dans le cas présent, nous ne retrouvons pas le fait que les moyennes obtenues à l'aide des deux modes de calcul sont très voisines l'une de l'autre et l'ensemble de nos résultats ne nous permet évidemment pas de savoir si l'une des méthodes que nous avons employées est meilleure que l'autre. Nous verrons plus loin les causes de cet échec.

De nombreuses expériences de digestibilité ont été effectuées sur Bovins par WAtson et ses collaborateurs (I945 à I949). Nous avons repris les valeurs trouvées par ces auteurs au cours d'une expérience réalisée en vue de connaître la digestibilité des betteraves ajoutées à une ration de base de foin, et nous les avons goupées 2 par 2 comme nous avons tenté de le faire par nos propres résultats. Nous trouvons ainsi des variations considérables des valeurs de la digestibilité, et de la ration de base (foin), et de l'aliment ajouté (betteraves). Aussi pensons-nous, à la suite de tous ces essais, qu'il n'y a pas lieu de substituer à la méthode classique de KELLNER, la nouvelle technique que nous avons proposée.

\section{DIGESTIBILITÉ DES DIFFÉRENTS COMPOSANTS DES DEUX MÉLANGES ALIMENT COMMERCIAL-SON ET ALIMENT COMMERCIAL-TOURTEAU DE SOJA}

\section{a) Mélange aliment commercial-son}

Nos résultats sont réunis dans le tableau de l'Annexe IV. Ils montrent que la digestibilité des matières organiques, des matières minérales, des matières cellulosiques, du phosphore et du calcium de la ration décroît au fur et à thesure que la proportion de son augmente dans le régime. Toutefois, seule la digestibilité du calcium est diminuée d'une façon statistiquement significative. $\left(t=3,5^{6}\right.$ pour les deux séries $\mathrm{V}$ et IV ; signification pour : 2,45$)$.

Par contre, la digestibilité des matières azotées reste pratiquement inchangée.

Nous retrouvons bien là le fait que le son laisse inchangée la digestibilité apparente des composés azotés alors qu'il fait diminuer 1'utilisation digestive des autres composants organiques du régime. Et nous ajoutons qu'il diminue également la digestibilité de la partie minérale du régime. Nous rejoignons ainsi les conclusions des chercheurs qui, dans la fameuse querelle de la farine entière contre la farine blutée, ont définitivement opté pour cette dernière.

La diminution de la digestibilité du $\mathrm{Ca}$ dans une ration contenant du son est due : $\mathbf{I}^{0}$ à la faible teneur en Calcium de cet aliment $(0, \mathrm{Ir} \%)$; quand la proportion de son dans le régime passe de o à $20 \%$, la teneur en calcium de la 
ration passe de $\mathrm{I}, 27$ à $\mathrm{I}, 08 \mathrm{p}$. Ioo g secs ; elle diminue donc de $15 \%$. Simultanément, il y a déséquilibre Phosphore-Calcium, le rapport $\mathrm{Ca} / \mathrm{P}$ variant de $\mathrm{I}, 33$ à $\mathrm{I}, 02$.

$2^{\circ}$ A la présence, dans le son, d'acide phytique dont on connaît bien l'action décalcifiante.

Il y aurait donc lieu de supplémenter en Calcium les aliments du bétail, dont la formule comporte une certaine proportion de son ou de rémoulages, tout comme on le fait pour les farines panifiables peu blutées, de façon à rétablir un apport normal de cet élément et à neutraliser l'action de l'acide phytique.

\section{b) Mélange aliment commercial-tourteau de soja (Annexe V)}

La digestibilité des composants organiques de la ration ne varie pratiquement pas après addition au régime de base de 5 à ro \% de tourteau de soja. Par contre, celle du Phosphore et du Calcium est soumise à des variations importantes. Compte tenu du fait que nous avons été obligés de changer d'aliment de base au cours de l'expérimentation, il semble que l'incorporation de $5 \%$ de tourteau de soja à une ration du commerce augmente la digestibilité du Ca et surtout celle du Phosphore (pour les séries VI et VII, la différence des digestibilités moyennes est significative), l'utilisation des matières minérales totales restant cependant inchangée. Il existerait donc, pour ce mélange alimentaire, un phénomène de "digestibilité associative " au moins pour le phosphore. Au-delà de $5 \%$, l'adjonction de soja à un aliment du commerce fait peù à peu disparaître cette "digestibilité associative ": pour 7,5\%,1'utilisation digestive du calcium et du phosphore est comparable à celle que l'on observe avec l'aliment de base seu1; pour to \%, elle est nettement inférieure dans le cas $\mathrm{du}$ calcium.

Les apports de Calcium et de Phosphore par l'alimentation ne peuvent rendre compte de ces variations ; les analyses des échantillons prélevés en cours d'expérience montrent, en effet, que les teneurs des rations en ces éléments sont très voisines les unes des autres pour les séries dans lesquelles le même aliment de base a été utilisé. Il y a donc lieu de supposer que la nature même de cet apport est telle qu'elle rend disponible pour l'organisme de plus grandes quantités de Calcium et de Phosphore lorsque le tourteau de soja entre dans l'alimentation du Lapin à raison de $5 \%$ de la ration totale.

Les valeurs groupées à l'Annexe $\mathrm{V}$ indiquent, en outre, que, pour les séries IX et $\mathrm{X}$ - aliment $\mathrm{n}^{0} 2$ seul ou additionné de $7,5 \%$ de soja - les digestibilités du $\mathrm{Ca}$ et $\mathrm{du} \mathrm{P}$ sont nettement différentes de celles que nous avons déterminées pour les séries précédentes, réalisées avec l'aliment $\mathrm{n}^{0} \mathrm{I}$ comme ration de base. Or la composition minérale des detix aliments n'est pas la même: pour l'aliment $\mathrm{n}^{\circ} \mathrm{I}$, le rapport $\mathrm{Ca} / \mathrm{P}$ est de $\mathrm{I}, 43$; pour $1 \mathrm{le} \mathrm{n}^{\circ} 2$, de $2, \mathrm{I} 8$. Il résulte de cette comparaison qu'un aliment pour Lapins doit contenir Calcium et Phosphore en quantités telles que le rapport de ces constituants soit nettement 
supérieur à 2 ; on arrive, dans ces conditions, à obtenir, pour les deux éléments, une digestibilité de l'ordre de $50 \%$. Il resterait bien entendu, à étendre ces recherches et à déterminer les limites inférieure et supérieure du rapport $\mathrm{Ca} / \mathrm{P}$ pour lesquelles la digestibilité des deux éléments reste satisfaisante, ainsi que les teneurs optima en $\mathrm{Ca}$ et $\mathrm{P}$ de la ration pour un rapport $\mathrm{Ca} / \mathrm{P}$ donné.

\section{DIGESTIBILITE DES DIFFÉRENTS COMPOSANTS D'UN ALIMENT AJOUTÉ AU RÉGIME DE BASE}

La méthode de KELLNER permet théoriquement de calculer la digestibilité des composants alimentaires d'un aliment - ici,son ou tourteau de soja ajouté à une ration dont on a étudié - préalablement - l'utilisation digestive.

Nous avons effectué ces calculs et nos résultats sont groupés dans le tableau IV ci-dessous.

\section{TABLEAU IV}

Digestibilité des composants alimentaires $d u$ son et $d u$ tourteau de soja (Valeurs moyennes)

\begin{tabular}{|c|c|c|c|c|c|c|c|}
\hline Séries & de l'aliment & $\begin{array}{l}\text { Matières } \\
\text { organiques }\end{array}$ & $\begin{array}{l}\text { Matières } \\
\text { minérales }\end{array}$ & $\begin{array}{l}\text { Matières } \\
\text { cellulo- } \\
\text { siques }\end{array}$ & $\begin{array}{c}\text { Matières } \\
\text { azotées }\end{array}$ & $\mathrm{P}$ & $\mathrm{Ca}$ \\
\hline $\begin{array}{l}\text { Io }^{\mathbf{C}} \text { Son } \\
\text { III. }\end{array}$ & & & & & & & \\
\hline $\begin{array}{l}\text { HI...... } \\
\text { III..... }\end{array}$ & $\begin{array}{l}10 \\
15\end{array}$ & $\begin{array}{l}5 \mathrm{I}, 3 \\
64,8\end{array}$ & $\begin{array}{r}32,6 \\
9,8\end{array}$ & $\begin{array}{r}-41,0 \\
-36,6\end{array}$ & $\begin{array}{r}110,2 \\
81,4\end{array}$ & $\begin{array}{l}43,0 \\
\text { II }, 6\end{array}$ & $\begin{array}{r}-710,7 \\
-\quad 830,6\end{array}$ \\
\hline IV... & 20 & 59,3 & 19,7 & 2,0 & 89,3 & $\mathrm{I} 4,2$ & $-842,5$ \\
\hline 告 Soja & & & & & & & \\
\hline $\begin{array}{l}\text { VII... } \\
\text { VIII... }\end{array}$ & 5 & I I 2,6 & 62,5 & 95,9 & 95,9 & 578,8 & 210,5 \\
\hline $\begin{array}{r}\text { VIII... } \\
\text { X... }\end{array}$ & IO & 86,5 & 20,3 & 69,3 & 83,7 & 44,0 & $-402,7$ \\
\hline & 7,5 & 68,3 & 47,4 & 10,9 & 74,3 & $-39,8$ & I $7 \mathrm{I}, \mathrm{O}$ \\
\hline
\end{tabular}

A part les valeurs obtenues pour l'utilisation digestive des matières organiques du son et, si l'on veut, pour les matières azotées du soja, l'ensemble des résultats est totalement incohérent.

Est-ce à dire que la technique que nous avons utilisée ne convient pas pour la mesure de la digestibilité des composants d'une ration ? Ce serait mettre en doute toutes les valeurs que l'on trouve dans la littérature et qui ont été obtenues d'après la méthode de KELLNER. N'est-ce pas plutôt que cette méthode doit être employée dans certaines conditions bien déterminées et que nous ne nous sommes pas placés dans ces conditions ? C'est ce que nous nous proposons de voir dans le paragraphe suivant.

\section{Discussion}

Le calcul de KELINER, nous l'avons vu, suppose que la digestibilité de le ration de base - et donc de ses constituants - ne varie pas lorsqu'on ajouta 
à cette ration l'aliment dont on veut connaître l'utilisation digestive. Nous avons d'ailleurs montré, dans la première partie de ce mémoire que cette hypothèse est parfaitement justifiée, au moins en ce qui concerne la matière sèche des aliments.

Mais le calcul de la digestibilité d'un composant quelconque d'un aliment nous oblige à utiliser deux coefficients d'utilisation digestive : 1'un d'eux est relatif à la digestibilité de la ration de base, l'autre, à la digestibilité du mélange ration de base-aliment à expérimenter.

$\mathrm{Si}$, en effet, nous appelons $\mathrm{A}$ et $\mathrm{B}$ les quantités d'un composant donné - disons, pour fixer les idées, des matières cellulosiques - respectivement fournies par la ration de base et par l'aliment ajouté à cette ration ; si nous appelons $d, x$ et $\mathrm{D}$ les valeurs des coefficients d'utilisation digestive de ce composant respectivement pour la ration de base, l'aliment ajouté et le mélange ration de base-aliment, nous pouvons écrire :

$$
\mathrm{Ad}+\mathrm{Bx}=(\mathrm{A}+\mathrm{B}) \mathrm{D}
$$

$d$ et $\mathrm{D}$ sont déterminés expérimentalement. On peut donc calculer facilement $x$.

\section{a) Erreurs possibles sur la valeur des coefficients de digestibilité}

Toutefois la mesure de la digestibilité d'une ration, par la pesée des ingesta et des excreta implique nécessairement un certain nombre d'erreurs : erreurs de pesée d'abord, et aussi et surtout, erreurs dues à 1' individualité " des animaux mis en expérience. L'excrétion n'est pas, jour après jour, rigoureusement proportionnelle à l'ingestion et l'on sait bien que les coefficients de digestibilité mesurés quotidiennement varient dans d'assez larges limites. C'est cet ensemble d'erreurs que traduit numériquement "l'erreur standard "sur le coefficient moyen d'utilisation digestive.

D'autre part, le calcul statistique montre que si la valeur déterminée expérimentalement ne s'écarte pas de la valeur réelle (que 1'on tirerait d'une infinité de mesures) de plus de son "erreur standard ", la différence entre valeur expérimentale et valeur réelle n'est pas significative $(t=\mathrm{I})$.

Il en résulte que lorsque nous disons : la digestibilité moyenne p. Ioo d'une ration est, $72,9 \pm \mathrm{I}, 3$, nous aurions pu trouver aussi bien 74,2\% ou $7 \mathrm{I}, 6 \%$, par le simple fait du hasard de "l'échantillonnage", si, par exemple, nous avions commencé les mesures sur les mêmes animaux, un ou deux jours plus tard.

Cette incertitude sur la valeur même du facteur que nous mesurons a des répercussions considérables sur le calcul de la méthode KELLNer. Pour ne prendre qu'un exemple, essayons de déterminer la digestibilité de la matière sèche du son ajouté à raison de ro \% à la ration de base. L'expérience nous a donné une digestibilité de 74,6 I I,6 pour la ration de base seule et de 73,0 士 I,4 pour le mélange. Si l'on effectue le calcul avec ce couple de valeurs 74,6 et 73,0 - on trouve que la matière sèche du son est utilisée par le Lapin à raison de $58,6 \%$. Mais nous pouvons aussi bien prendre, comme bases de 
calcul, les 2 couples de valeurs $74,6+\mathrm{r}, 6$ et $73,0-\mathrm{I}, 3$ et $74,6-\mathrm{I}, 6$ et 73,0 $+\mathrm{I}, 3$. Dans ces conditions, la digestibilité moyenne du son dans notre expérience est ou bien 3I \% ou bien $86 \%$.

\section{b) Conditions d'emploi de la méhode de KELINER}

Pour lever pareille incertitude, il n'y a qu'une seule solution: il faut diminuer autant que possible la valeur de "l'erreur standard" sur la moyenne. Eit, pour ce faire, nous ne disposons que de 2 moyens : augmenter le nombre des déterminations et diminuer les écarts à la moyenne, c'est-à-dire expérimenter sur des animaux parfaitement en régime.

Il est possible que, dans notre expérimentation, la durée de l'adaptation aux rations, ainsi que la durée même des mesures, aient été trop faibles. Mais l'étude des coefficients d'utilisation digestive mesurés jour après jour met en évidence, particulièrement chez certains animaux (lapins $\mathrm{n}^{\circ} 2,3$ et 6 notamment), des variations importantes que nous pensons pouvoir expliquer de la façon suivante. L'aliment de référence que nous avons utilisé est hautement digestible ; c'est, en fait, un aliment " complémentaire " qui, dans la pratique, doit être fourni aux Lapins en " complément " d'une ration d'aliments encombrants. Nous l'avons utilisé seul ou en mélange avec des rations de "concentrés ". Il en résulte que, d'une part, l'excrétion fécale est faible - de l'ordre de I6$20 \mathrm{~g}$ secs en moyenne par animal et par jour - et que, par suite, les erreurs de pesées prennent davantage d'importance; d'autre part, que le tractus intestinal ne peut se mettre en régime à cause du trop faible volume du bol alimentaire. Il ne faut pas oublier, en effet, que, chez un lapin de $3 \mathrm{~kg}$ environ, la longtueur de l'intestin dépasse $3 \mathrm{~m}$ et qu'il est nécessaire, pour obtenir un fonctionnement régulier de l'appareil digestif que l'encombrement de la ration soit suffisant. Dans nos essais, le coefficient d'encombrement des régimes utilisés (matière sèche /unités fourragères) variait de I à I, I5. Et d'après de nombreuses déterminations, nous sommes en mesure de dire que la dispersion des valeurs quotidiennes du coefficient d'utilisation digestive chez le lapin est considérablement réduite si le coefficient d'encombrement de la ration atteint 1,25 .

\section{c) Erreurs dues a l'analyse chimique}

Tout ce qui précède nous permet de comprendre pourquoi nous n'avons pu arriver à des résultats cohérents lorsqu'il s'est agi de déterminer la digestibilité des composants des aliments ajoutés à la ration de base.

Les coefficients d'utilisation digestive de ces composants sont entachés de la même erreur que ceux relatifs à la matière sèche - ceux-ci étant les seules valeurs déterminées expérimentalement. A cette erreur s'ajoutent, bien entendu, les inévitables erreurs de l'analyse chimique (Cf. Erreur commise dans le calcul de la valeur fourragère d'un aliment composé en fonction des erreurs de l'Analyse chimique par A. Françors (r949). Mais, à la différence du cas 
précédent, nous ne pouvons ici déterminer la marge de variation possible des valeurs calculées : nous ne possédons, en effet, qu'une seule analyse des ingesta et des excreta par série expérimentale. Toujours est-il que cette marge est nécessairement encore plus importante que celle dont nous avons pu montrer l'existence pour la détermination de la digestibilité de la matière sèche d'une ration.

Pour éviter ces inconvénients, il nous semble que la solution consiste à réaliser des analyses d'ingesta et d'excreta le plus souvent possible au cours des séries expérimentales, de façon à être en mesure de déterminer la part qui revient à l'analyse chimique dans les erreurs des déterminations.

\section{CONCLUSIONS}

$I^{0}$ Lorsque l'on ajoute à une ration commerciale pour Lapins, soit du son (Io à $20 \%$ ), soit du tourteau de soja ( 5 à Io \%), la digestibilité de la matière sèche de cette ration commerciale ne varie pratiquement pas, en moyenne.

$2^{\circ}$ La digestibilité de la matière sèche de la ration totale diminue légèrement lorsque le régime renferme du son; elle ne varie pratiquement pas lorsque le régime renferme du tourteau de soja.

$3^{\circ}$ La détermination de la digestibilité d'un aliment au moyen de la méthode classique de KELINER, donne des résultats moins dispersés que la méthode qui consiste à grouper les résultats de deux séries expérimentales et à calculer séparément les digestibilités des deux composants principaux de la ration.

$4^{0}$ Lorsqu'un régime pour Lapins contient une forte proportion de son (Io à $20 \%$ ), seule la digestibilité apparente des matières azotées de la ration reste inchangée; la digestibilité du Calcium est considérablement diminuée. D'où la nécessité d'incorporer à un tel régime des suppléments de sels de calcium.

Si le régime renferme de 5 à ro \% de tourteau de soja, la digestibilité des matières organiques ne varie pratiquement pas; celle du Phosphore et du Calcium dépend de la proportion de tourteau dans la ration.

$5^{\circ}$ Pour qu'un régime destiné à l'alimentation du Lapin permette une utilisation digestive de 1'ordre de $50 \%$ du Calcium et du Phosphore, il faut que le rapport $\mathrm{Ca} / \mathrm{P}$ soit au minimum égal à 2 .

$6^{\circ}$ Il n'a pas été possible d'obtenir des valeurs cohérentes des différents composants alimentaires des aliments (son et tourteau de soja) ajoutés à la ration de base. La discussion des causes de cet échec met en évidence les faits suivants qui conditionnent l'emploi de la méthode de KELLNER lorsqu'on veut se servir du Lapin comme animal d'expérience. Pour pouvoir utiliser avec certitude ce mode de calcul, il faut :

- Expérimenter sur des animaux parfaitement adaptés au régime et dont les régulations physiologiques soient aussi précises que possible - ce qui implique la nécessité d'éliminer certains sujets, même lorsqu'on dispose d'animaux sélectionnés.

- Disposer d'un nombre aussi grand que possible de déterminations pour 
atténuer, dans toute la mesure souhaitable, les variations dues à l'individualité même des animaux. La durée des mesures doit être d'autant plus longue que les écarts que l'on observe, d'un jour à l'autre, sont plus importants.

- Utiliser un aliment de référence suffisamment encombrant (coefficient d'encombrement au moins égal à $I, 2$ ) pout que l'excrétion fécale soit importante et aussi régulière que possible.

- Réaliser, au cours des séries expérimentales, plusieurs déterminations de la composition chimique des ingesta et des excreta pour pouvoir évaluer l'erreur possible due aux analyses elles-mêmes.

\section{ANNEXE I}

Composition \% des aliments utilisés dans les différentes séries expérimentales

\begin{tabular}{|c|c|c|c|c|}
\hline & \multicolumn{2}{|c|}{$\begin{array}{l}\text { Aliments du } \\
\text { commerce } \\
\text { pour lapins }\end{array}$} & & \multirow[b]{2}{*}{ Tourteau } \\
\hline & $\mathrm{n}^{0} \mathrm{I}$ & $\begin{array}{c}n^{0} 2 \\
-\end{array}$ & Son & \\
\hline Humidité $\ldots \ldots \ldots \ldots \ldots \ldots \ldots \ldots \ldots \ldots \ldots \ldots \ldots \ldots \ldots \ldots$ & 11,30 & 10,49 & I 2, I 3 & 10,91 \\
\hline 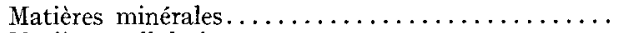 & 6,67 & 7,80 & 5,52 & 7,00 \\
\hline Matières cellulosiques $\ldots \ldots \ldots \ldots \ldots \ldots \ldots \ldots \ldots$ & $8,3 \mathbf{i}$ & 9,68 & IO, I 2 & $3,3 \mathrm{I}$ \\
\hline Matières azotées $\ldots \ldots \ldots \ldots \ldots \ldots \ldots \ldots \ldots \ldots$ & 17,96 & 16,78 & I 5,73 & 47,46 \\
\hline Matières grasses $\ldots \ldots \ldots \ldots \ldots \ldots \ldots \ldots \ldots$ & 3,27 & 4,67 & 5,04 & 3,53 \\
\hline Extractif non azoté $\ldots . \ldots \ldots \ldots \ldots$ & 52,49 & 50,58 & 51,46 & 27,79 \\
\hline Unités fourragères $\% \mathrm{~kg} \ldots \ldots \ldots \ldots \ldots \ldots$ & 83 & 78 & 75 & II I \\
\hline Matières azotées digestibles. & I 3,8 & $x 2,4$ & I $3, I$ & 43,6 \\
\hline Matière sèche & 88,70 & $89,5 \mathrm{I}$ & 87,87 & 89,09 \\
\hline Acidité $\left(\mathrm{g} \mathrm{SO}_{4} \mathrm{H}_{2}\right)$ & 0,44 & 0,25 & 0,35 & O,I 5 \\
\hline$\ldots \ldots \ldots \ldots \ldots \ldots \ldots$ & 0,80 & 0,70 & 1,26 & 0,92 \\
\hline Calcium.. . & $\mathrm{I}, \mathrm{I} 4$ & 1,52 & o, I I & 0,29 \\
\hline
\end{tabular}

ANNEXE II

Données expérimentales pour les calculs de digestibilité du mélange son-farine Ingestion - Excrétion - Absorption

\begin{tabular}{|c|c|c|c|c|c|}
\hline Numéros des lapins & 2 & 3 & 4 & 5 & Totaux \\
\hline $\begin{array}{r}\text { Série } V: \text { Aliment } n^{\circ} \mathrm{r} \text {, } \\
\text { roo } \%: \\
\text { Ingesta } \ldots \ldots \ldots \ldots \ldots \ldots \\
\text { Excreta } \ldots \ldots \ldots \ldots \ldots \ldots \\
\text { Absorption } \ldots \ldots \ldots \ldots \ldots \\
\end{array}$ & $\begin{array}{l}809,0 \\
210,4 \\
598,6\end{array}$ & $\begin{array}{r}588,5 \\
97,9 \\
490,6\end{array}$ & $\begin{array}{l}939,5 \\
265,7 \\
673,8\end{array}$ & $\begin{array}{l}755,4 \\
210,6 \\
544,8\end{array}$ & $\begin{array}{c}3092,4 \\
784,6 \\
2307,8\end{array}$ \\
\hline $\begin{array}{l}\text { Série } I I: \text { Aliment } \mathrm{n}^{\circ} \mathrm{I} \\
++ \text { Io } \% \text { de son : } \\
\text { Ingesta } \ldots \ldots \ldots \ldots \ldots \ldots \\
\text { Excreta. } \ldots \ldots \ldots \ldots \ldots \\
\text { Absorption } \ldots \ldots \ldots \ldots \ldots \\
\end{array}$ & $\begin{array}{l}624,6 \\
167,6 \\
457,0\end{array}$ & $\begin{array}{l}474,3 \\
\text { I1 } 3,9 \\
360,4 \\
\end{array}$ & $\begin{array}{l}971,4 \\
264,2 \\
707,2\end{array}$ & $\begin{array}{l}660,5 \\
188,6 \\
471,9\end{array}$ & $\begin{array}{r}730,8 \\
734,3 \\
\text { I } 996,5 \\
\end{array}$ \\
\hline $\begin{array}{l}\text { Série } 111: \text { Aliment } \mathrm{n}^{\circ} \text { I } \\
\quad+\text { I } 5 \% \text { de son : } \\
\text { Ingesta } \ldots \ldots \ldots \ldots \ldots \ldots \\
\text { Excreta } \ldots \ldots \ldots \ldots \ldots \ldots \\
\text { Abcorption } \ldots \ldots \ldots \ldots\end{array}$ & $\begin{array}{l}642,0 \\
\mathrm{I} 78,8 \\
463,2\end{array}$ & $\begin{array}{l}539, \text { I } \\
\text { I } 35,4 \\
403,7\end{array}$ & $\begin{array}{l}843,0 \\
265,1 \\
577,9\end{array}$ & $\begin{array}{l}746,3 \\
217,8 \\
528,5\end{array}$ & $\begin{array}{r}2770,4 \\
797, \mathrm{r} \\
\text { I } 973,3\end{array}$ \\
\hline $\begin{array}{l}\text { Série } I V: \text { Aliment } \mathrm{n}^{\mathrm{o}} \\
\quad+20 \% \text { de son : } \\
\text { Ingesta } \ldots \ldots \ldots \ldots \ldots \ldots \\
\text { Excreta. } \ldots \ldots \ldots \ldots \ldots \ldots \\
\text { Absorption } \ldots \ldots \ldots \ldots\end{array}$ & $\begin{array}{r}728,9 \\
201,0 \\
527,9\end{array}$ & $\begin{array}{l}647,3 \\
\mathbf{I} 88,3 \\
459,0\end{array}$ & $\begin{array}{l}937,9 \\
278,0 \\
659,9\end{array}$ & $\begin{array}{l}745,6 \\
223,5 \\
522,1\end{array}$ & $\begin{array}{r}3059,7 \\
890,8 \\
2 \mathrm{I} 68,9\end{array}$ \\
\hline
\end{tabular}




\section{ANNEXE III}

Données expérimentales pour les calculs de digestibilité du mélange soja-farine Ingesta - Excreta - Absorption

\begin{tabular}{|c|c|c|c|c|c|c|}
\hline Numéros des lapins & 6 & 7 & 8 & 9 & 10 & Totaux \\
\hline 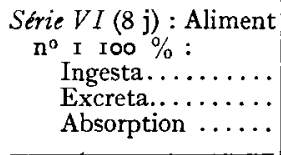 & $\begin{array}{r}452,7 \\
97,8 \\
354,9\end{array}$ & $\begin{array}{l}718,2 \\
174,8 \\
543,4\end{array}$ & $\begin{array}{r}373,5 \\
79,9 \\
293,6\end{array}$ & $\begin{array}{l}533,0 \\
\text { I I } 8,5 \\
4 I 4,5\end{array}$ & $\begin{array}{l}642,8 \\
\text { I } 71,0 \\
471,8\end{array}$ & $\begin{array}{r}2720,2 \\
642,0 \\
2078,2\end{array}$ \\
\hline 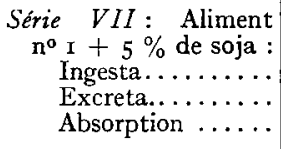 & $\begin{array}{l}807,8 \\
163,4 \\
644,4\end{array}$ & $\begin{array}{l}844,2 \\
202,4 \\
64 \mathrm{I}, 8\end{array}$ & $\begin{array}{l}649,0 \\
\mathrm{I} 37, \mathrm{I} \\
5 \mathrm{II}, 9\end{array}$ & $\begin{array}{l}766,1 \\
169,9 \\
596,2\end{array}$ & $\begin{array}{l}697,8 \\
151,2 \\
546,6\end{array}$ & $\begin{array}{r}3764,9 \\
824,0 \\
2940,9\end{array}$ \\
\hline $\begin{array}{r}\text { Série VIII : Aliment } \\
\mathbf{n}^{\mathbf{0}} \mathbf{r}+\mathbf{r o} \% \text { de soja : } \\
\text { Ingesta............ } \\
\text { Excreta........... } \\
\text { Absorption ...... }\end{array}$ & $\begin{array}{l}688,5 \\
\mathrm{r} 44,3 \\
544,2\end{array}$ & $\begin{array}{l}816,0 \\
214,3 \\
601,7\end{array}$ & $\begin{array}{l}620,0 \\
140,7 \\
479,3\end{array}$ & $\begin{array}{l}644,7 \\
\text { r } 43,7 \\
501,0\end{array}$ & $\begin{array}{r}430,7 \\
95,9 \\
334,8\end{array}$ & $\begin{array}{r}3199,9 \\
738,9 \\
2461,0\end{array}$ \\
\hline $\begin{array}{c}\text { Série IX : Aliment } \mathrm{n}^{0} 2 \\
\text { Ioo } \%: \\
\text { Ingesta............. } \\
\text { Excreta........... } \\
\text { Absorption ...... }\end{array}$ & $\begin{array}{l}6 \mathrm{I} 8,8 \\
\mathrm{I} 75,6 \\
443,2\end{array}$ & $\begin{array}{l}929,7 \\
249,8 \\
679,9\end{array}$ & $\begin{array}{l}702,0 \\
191,3 \\
510,7\end{array}$ & $\begin{array}{l}936,6 \\
254,0 \\
682,6\end{array}$ & $\begin{array}{l}874,7 \\
230,6 \\
644, \mathrm{I}\end{array}$ & $\begin{array}{l}4 \text { O6I, } 8 \\
\text { I IOI,3 } \\
2960,5\end{array}$ \\
\hline $\begin{array}{r}\text { Série } X: \text { Aliment } \mathrm{n}^{0}{ }^{2} \\
+\quad 7,5 \% \text { de soja } \\
\quad \text { Ingesta........... } \\
\quad \text { Excreta } \ldots \ldots \ldots \ldots \\
\quad \text { Absorption } \ldots \ldots \ldots\end{array}$ & $\begin{array}{l}735, \mathrm{I} \\
200,0 \\
535, \mathrm{I}\end{array}$ & $\begin{array}{l}780,0 \\
219, \circ \\
561,0\end{array}$ & $\begin{array}{l}777,0 \\
210,6 \\
566,4\end{array}$ & $\begin{array}{l}847,5 \\
233,3 \\
614,2\end{array}$ & $\begin{array}{l}7 \mathrm{I} 7, \mathrm{I} \\
200,7 \\
5 \mathrm{I} 6,4\end{array}$ & $\begin{array}{l}3856,7 \\
1063,6 \\
2 \quad 793,1\end{array}$ \\
\hline
\end{tabular}

ANnEXE IV

Digestibilités brutes moyennes et individuelles des différents composants nutritifs de la ration

\begin{tabular}{|c|c|c|c|c|c|c|}
\hline \multirow{2}{*}{ Séries } & \multirow{2}{*}{$\%$ de son } & \multicolumn{4}{|c|}{ Numéros des lapins } & \multirow{2}{*}{ Moyennes } \\
\hline & & 2 & 3 & 4 & 5 & \\
\hline \multicolumn{7}{|c|}{$I^{\circ}$ Matière organique : } \\
\hline 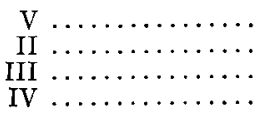 & $\begin{array}{l}0 \\
10 \\
15 \\
20\end{array}$ & $\begin{array}{l}75,7 \\
74,9 \\
74,0 \\
74,1\end{array}$ & $\begin{array}{l}84,6 \\
77,5 \\
76,4 \\
72,9\end{array}$ & $\begin{array}{l}73,0 \\
74,5 \\
7 \mathrm{I}, 4 \\
7 \mathrm{I}, 9\end{array}$ & $\begin{array}{l}73,8 \\
68,4 \\
72,6 \\
7 \times, 9\end{array}$ & $\begin{array}{l}76,1 \\
73,6 \\
73,3 \\
72,7\end{array}$ \\
\hline \multicolumn{7}{|c|}{ II' Matières minérales : } \\
\hline 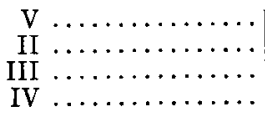 & $\begin{array}{l}0 \\
\text { I0 } \\
\text { I } 5 \\
20\end{array}$ & $\begin{array}{l}5 \mathrm{I}, 9 \\
5 \mathrm{I}, 4 \\
48,0 \\
49,7\end{array}$ & $\begin{array}{l}67,5 \\
57,6 \\
55,4 \\
44,7\end{array}$ & $\begin{array}{l}44,9 \\
5 \mathrm{I}, 5 \\
3 \mathrm{I}, 8 \\
48,8\end{array}$ & $\begin{array}{l}51,4 \\
45,2 \\
48,2 \\
44,6\end{array}$ & $\begin{array}{l}52,6 \\
50,9 \\
44,6 \\
46,9\end{array}$ \\
\hline
\end{tabular}


ANNEXE IV (suite)

\begin{tabular}{|c|c|c|c|c|c|c|c|}
\hline \multirow{2}{*}{\multicolumn{2}{|c|}{ Séries }} & \multirow{2}{*}{$\%$ de son } & \multicolumn{4}{|c|}{ Numéros des lapins } & \multirow{2}{*}{ Moyenne } \\
\hline & & & 2 & 3 & 4 & 5 & \\
\hline \multicolumn{8}{|c|}{ IIIo Matières cellulosiques : } \\
\hline $\mathrm{V}$ & $\ldots \ldots \ldots \ldots \ldots$ & o & 30,7 & 52,5 & 20,9 & $3^{2,2}$ & 32,3 \\
\hline II & $\ldots \ldots \ldots \ldots \ldots$ & Io & 23,4 & 30,8 & 21,5 & $2 \mathrm{I}, 2$ & 23,5 \\
\hline III & $\ldots \ldots \ldots \ldots \ldots$ & I5 & $20, \mathrm{I}$ & 26,1 & I $3, \mathrm{I}$ & 23,2 & 20,0 \\
\hline IV & $\ldots \ldots \ldots \ldots \ldots$ & 20 & 23,5 & 24,6 & 25,6 & 26,9 & 25,2 \\
\hline \multicolumn{8}{|c|}{ IVo Matières protéiques: } \\
\hline $\mathrm{V}$ & $\ldots \ldots \ldots \ldots \ldots$ & 0 & 72,2 & 85,3 & 70,0 & 69,5 & 73,3 \\
\hline II & $\ldots \ldots \ldots \ldots \ldots$ & Io & 77,7 & 83,3 & 74,8 & 73,4 & 76,6 \\
\hline III & $\ldots \ldots \ldots \ldots \ldots$ & I5 & 74,4 & 77,8 & 70,8 & 75,9 & 74,4 \\
\hline IV & $\ldots \ldots \ldots \ldots \ldots$ & 20 & 75,2 & 78,6 & 74,0 & 78,0 & 76,2 \\
\hline \multicolumn{8}{|c|}{ Vo Phosphore: } \\
\hline $\mathrm{V}$ & $\ldots \ldots \ldots \ldots \ldots$ & 0 & - & $6 I, 4$ & 25,0 & $3^{6,3}$ & $38, \mathrm{r}$ \\
\hline II & $\ldots \ldots \ldots \ldots \ldots$ & Io & 37,3 & 44,6 & 39,8 & 35,2 & 38,9 \\
\hline III & $\ldots \ldots \ldots \ldots \ldots \ldots$ & I 5 & 35,5 & 43,0 & 20,7 & 34,6 & 32,2 \\
\hline IV & $\ldots \ldots \ldots \ldots \ldots$ & 20 & $3^{6,7}$ & 30,5 & 28,8 & 30,1 & $3 r, 3$ \\
\hline \multicolumn{8}{|c|}{ VIo Calcium : } \\
\hline $\mathrm{V}$ & $\ldots \ldots \ldots \ldots \ldots$ & $\circ$ & $6 \mathrm{r}, 7$ & 66,0 & 43,4 & 54,7 & 55,3 \\
\hline II & $\ldots \ldots \ldots \ldots$ & IO & 45,2 & $49, \mathrm{I}$ & 47,2 & 48,3 & 47,4 \\
\hline III & $\ldots \ldots \ldots \ldots \ldots$ & I 5 & 42,5 & 47,5 & $3 \mathrm{I}, 2$ & 46,0 & 41,0 \\
\hline IV & $\ldots \ldots \ldots \ldots \ldots$ & 20 & $44, \mathrm{I}$ & $3^{\mathrm{I}}, 5$ & 28,7 & $3^{6,5}$ & 34.9 \\
\hline
\end{tabular}

ANNEXE V

Digestibilités brutes moyennes et individuelles des différents composants nutritifs de la ration

Régime : Aliment commercial + Tourteau de Soja

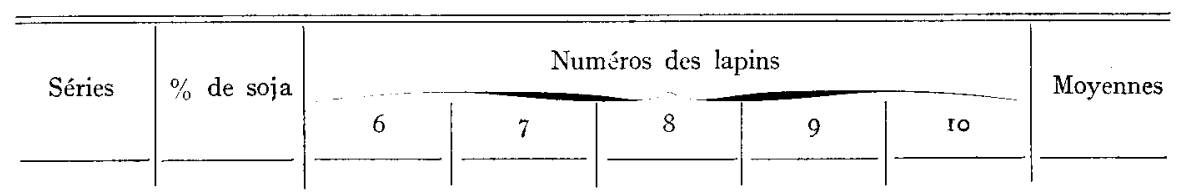

$I^{0}$ Matières organiques:

\begin{tabular}{|c|c|c|c|c|c|c|c|}
\hline VI. .... & 0 & 80,5 & 77,7 & 80,4 & 79,8 & 76,0 & 78,5 \\
\hline VII.... & I 5 & 80,4 & 80,0 & $8 \mathrm{I}, \mathrm{O}$ & $78, \mathrm{I}$ & $8 \mathrm{I}, 9$ & 80,2 \\
\hline VIII..... & IO & $8 \mathrm{r}, 4$ & $76, \mathbf{I}$ & 79,8 & $80, I$ & 80,3 & 79,3 \\
\hline IX.. & 0 & 73,8 & 75,3 & 74,9 & 74,8 & 75,5 & 74,9 \\
\hline X. & 7,5 & $75, \mathbf{I}$ & 73,9 & 74,5 & 74,6 & $74, \mathrm{I}$ & 74,4 \\
\hline
\end{tabular}

IIo Matières minérales :

\begin{tabular}{|c|c|c|c|c|c|c|c|}
\hline VI.... & 0 & 52,9 & $5^{I, 6}$ & 58,2 & 53,2 & 42,9 & 50,9 \\
\hline VII..... & 5 & 53,4 & 50,0 & 52,2 & 50,0 & $52, I$ & $5 \mathrm{x}, 5$ \\
\hline VIIT..... & Io & $5 \mathrm{I}, \mathrm{I}$ & 44,6 & 46,8 & 49,3 & 46,8 & 47,7 \\
\hline IX. & 0 & 48,0 & 49,5 & 48,5 & $5 \mathrm{r}, \mathrm{I}$ & 53,3 & 50,3 \\
\hline X. & 7,5 & 47,2 & 49,9 & 54,7 & 49,3 & 49,2 & $50, I$ \\
\hline
\end{tabular}

III Matières cellu'osiques :

\begin{tabular}{r|c|l|l|l|l|l|l} 
VI..... & 0 & 25,2 & 25,0 & 36,3 & 29,8 & 20,5 & 28,9 \\
VIII.... & 5 & 38,8 & 23,0 & 29,0 & $27, \mathrm{I}$ & 34,0 & 30,3 \\
VIII..... & I0 & 34,4 & 25,0 & 26,6 & $33, \mathrm{I}$ & 37,3 & 30,6 \\
IX.... & 0 & 23,05 & 30,8 & 29,5 & 34,4 & 26,7 & 29,3 \\
X.... & 7,5 & $3 \mathrm{I}, 3$ & 28,2 & $28, \mathrm{r}$ & 30,3 & 25,6 & 28,8 \\
\hline
\end{tabular}


ANNEXE V (suite)

Séries $\mid \%$ de soja $\left|\begin{array}{c|c|c|c|}\text { Numéros des lapins } & \text { I0 } \\ \hline\end{array}\right|$

IVo Matières protéiques :

\begin{tabular}{r|c|l|l|l|l|l|l} 
VI..... & o & 83,7 & 80,7 & 84,0 & 83,5 & 79,4 & 81,9 \\
VII...... & 5 & 85,5 & 80,9 & 84,8 & 84,6 & 82,4 & 83,6 \\
VIII...... & Io & 84,4 & 77,3 & 85,0 & 83,6 & 82,9 & 82,3 \\
IX.... & $\circ$ & 86,9 & 83,2 & 84,6 & 83,4 & 87,4 & 85,0 \\
X..... & 7,5 & 87,0 & 78,8 & 84,6 & 81,7 & 83,1 & 83,0
\end{tabular}

Vo Matières grasses :

\begin{tabular}{ll|l|l|l|l|l|l}
$\mathrm{IX} . . .$. & 0 & 85,2 & 80,9 & 83,2 & 83,8 & 84,9 & 83,5 \\
$\mathrm{X} . . .$. & 7,5 & 82,4 & 80,8 & $8 \mathrm{I}, 3$ & 81,0 & 82,5 & $8 \mathrm{r}, 6$
\end{tabular}

VIo Extractif non azoté :

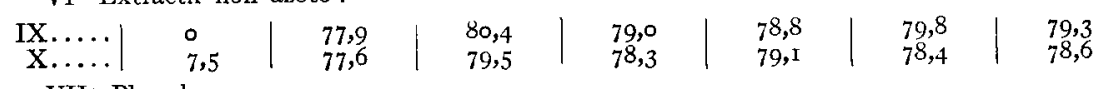

VII' Phosphore :

\begin{tabular}{|c|c|c|c|c|c|c|c|}
\hline V1. & o & 27,9 & 25,6 & 32,0 & 28,0 & 10,0 & 23,7 \\
\hline VII..... & 3 & 54,2 & 58,8 & 56,0 & 54,9 & 46,3 & 54,2 \\
\hline VIII. & I0 & 29,7 & 21,5 & 22,8 & $29, \mathrm{I}$ & 28,0 & 25,9 \\
\hline IX..... & 0 & 35,6 & 47,4 & 53,2 & 46,9 & 46,5 & 46,3 \\
\hline $\mathrm{X}$. & 7,5 & 35,8 & $4 \mathbf{I}, 9$ & 30,2 & 48,4 & 30,8 & 37,7 \\
\hline
\end{tabular}

vIIIo Calcium :

\begin{tabular}{|c|c|c|c|c|c|c|c|}
\hline VI. & $\circ$ & 43,8 & 46,3 & 49,9 & 43,4 & 27,5 & $4^{1,4}$ \\
\hline VII..... & 5 & 46,4 & 46,3 & 46,9 & 40,8 & 39,9 & $44, \mathrm{I}$ \\
\hline VIII. . & Io & $3^{6,6}$ & 30,1 & 28,7 & 28,8 & 24,5 & $3^{\circ}, 2$ \\
\hline IX. & o & 47,0 & 50,7 & 55,7 & 54,0 & 50,0 & $5^{1,6}$ \\
\hline $\mathrm{X}$. & 7,5 & 47,7 & 54,4 & 61,0 & 53,9 & 47,6 & $53, \mathrm{I}$ \\
\hline
\end{tabular}

